\title{
Patients with dementia and depression-complexity
}

\author{
Samuel Langhorne* and Louisa May Alcott
}

*Corresponding Author: Samuel Langhorne. Department de Neurosciences Fondamentales,Cuba.Email:Iangorne_samuel32@gmail.edu

Received date: April 16,2018 ;Accepted date : May 26,2018; Published date: June 11,2018.

Citation : Samuel Langhorne, Patients with dementia and depression-complexity. J Psychology and Mental Health Care. Doi: $10.31579 / 2637-8892 / 030$

Copyright : () 2018 Samuel Langhorne. This is an open-access article distributed under the terms of The Creative Commons Attribution License, which permits unrestricted use, distribution, and reproduction in any medium, provided the original author and source are credited. Abstract

For a long time researchers and clinicians have been trying to clarify the possible existing relationships between Alzheimer's disease and the depression in the elderly. This article tries to take stock of these complex links. It seems that often depression is clinically confused with apathy.

\section{Introduction}

A recent article describes the body of evidence suggesting that depression earlier in life is a risk factor for problematic brain / cognitive aging ("dementia") and that depression in older adults may be a precursor [1]. Based on these data, it has been estimated that 10$15 \%$ of "Alzheimer's" cases are attributable to depression and that a $25 \%$ reduction in the prevalence of depression could lead to a decrease of 827.000 cases of "Alzheimer's disease" in the world and 173000 cases in the United States [2]. Moreover, it has been shown also show that depression occurs in 20 to $30 \%$ of people diagnosed with "Alzheimer's disease" and that this percentage is even higher in people diagnosed with "dementia vascular" and "Lewy body dementia" [3]. Finally, the researchers indicate that the mechanisms involved in the relationships between "dementia" and "depression" remain unknown and are likely multiple. They further add that there is no convincing evidence of the effectiveness of antidepressants in people with "dementia".

A better understanding of the links between depression and cerebral aging / cognitive seems imperative to us to go through the abandonment of a biomedical and categorical approach, both cerebral aging and depression. Once again, it is a question of adopting a continuum perspective, taking into account the complexity of the factors involved in these relationships.

\section{Depressive manifestations differ depending on the context}

While psychiatry is tending more and more to offer explanations at the molecular or neuronal level, the diversity of psychopathological symptoms and in particular the symptoms grouped under the term "depression", there is evidence that these symptoms depend significantly on psychological, environmental or cultural context. Disparities appear to be related to several methodological factors. The studies do not examine all the same populations. Thus, those carried out in psychiatry, neurology or general population do not recruit the same type of patients [4]. Moreover, within a "psychiatric" or "neurological" population, hospitalized patients generally have more severe or more chemo-resistant pathologies than those supported in ambulatory. Neither should the skills specific to each specialist and all the studies do not call on a panel of experts. Indeed, although having notions of psychiatry and using diagnostic criteria or even scales of evaluation a neurologist will be less able to diagnose atypical cases of depression, as a psychiatrist will be struggling with some patients who may have dementia symptoms. Not all researchers use the same definitions for pathologies, sometimes ending with to study different diseases and make it impossible to compare the results. First, it is important to define what is meant by "depression" and "dementia" [5].

Auctores Publishing - Volume1-10030 www.auctoresonline.org Page - 01
Indeed, some authors consider the major depressive episode, others subsyndromic depressions, others still depressive symptoms. This is increased in the study of depression in the elderly subject by compared to the young adult since depression is frequently atypical and can take different aspects as we have seen previously. In addition, many authors before the fact that subsyndromic episodes, often have as much impact as the major depressive episode on functional disability and cognition. This is even more obvious in the definition of cognitive disorders. Some researchers study the Mild Cognitive Impairment, others the cognitive decline, still others the dementia disorder. Yet, the etiology of dementia is not always specified [4]. When it is, some studies deal with the association between depression and dementia in general, others between depression and vascular dementia, still others between depression and Alzheimer's dementia, etc. Finally, the terms "dementia" and "Alzheimer's disease" are sometimes confused, the large proportion of dementias Alzheimer's have contributed to these terms being sometimes seen as interchangeable [6]. Even though the authors would strictly study the same pathologies, diagnoses are not always posed according to the same criteria. They give way to a certain subjectivity and do not are not as reliable as for some other diseases (for which it is for example possible biological markers), especially when investigating potential previous episodes, or to pinpoint the date of onset of the disorder [7]. Some authors using a categorical approach, the diagnosis is then established following a clinical examination, by an expert, according to recognized diagnostic criteria (usually DSM or ICD-10 criteria). Other authors have a dimensional approach, the diagnosis then relies on the use of more scales or less specific, used in autoor hetero-questionnaire [4]. Researchers hypothesize that, for depression, non-psychiatric practitioners do not follow the diagnostic criteria as well as psychiatrists (especially with regard to the minimum duration of symptoms in order to make the diagnosis) [6]. Others have noticed that among the authors studying the link between the depression of the elderly and dementia, those specialized in depression of the elderly subject tended to use the Hamilton Depression Rating Scale and the Geriatric Depression Scale as Depression Scale, while those specializing in dementia used the Neuropsychiatric Inventory and Cornell Scale for Depression in Dementia [8].

\section{Positive association between depression and dementia}

In a meta-analysis, all studies dealing with the relationship between depression and Alzheimer's disease were examined [9]. Including the studies to calculate a report Odds Ratio (OR) for risk of developing Alzheimer's disease or Dementia close to Alzheimer's disease in subjects with a history of depression by comparison with those without a history of depression. Studies that included patients with a diagnosis of vascular dementia and those whose diagnostic criteria for depression or Alzheimer's disease were vague were excluded. 
Twenty studies meeting these criteria were selected (out of 153 potential), representing 102,172 participants from eight different countries, each meeting the Newcastle Ottawa quality criteria. The results of this meta-analysis show that there is a relationship between a history of depression and the occurrence of Alzheimer's disease with OR of 2.03 [95\% CI 1.73-2.38] for case-specific c investigations and 1.90 [95\% CI 1.55-2.33] for cohort studies; a history of depression doubles the risk of developing Alzheimer's dementia [9].

Another meta-analysis of clinical studies, examining the relationship between depression and dementia (Alzheimer's disease and other causes of dementia) [10]. Fourteen clinical studies were selected: seven cohort studies and seven casecontrol surveys. The results are superimposable to those observed by [9], showing an association between depression and dementia (OR 1.87 [95\% CI 1.09-3.20] for cohort studies, OR 2.01 [95\% CI 1, 16-3.50] for case-control surveys). More recently, another meta-analysis examining the relationship between elderly depression and dementia, performing separate analyzes according to the type of dementia (Alzheimer's disease, vascular dementia and other rarer dementias) [11].

A total of 23 clinical studies were included on 1839 literature references; this represented a pool of 49,612 subjects $(5,116$ with depression and 44,496 non-depressive control subjects). The metaanalysis was based on 28,746 participants for Alzheimer's disease (3437 depressed subjects, 25,309 controls) and 14,901 participants for vascular dementia (1801 depressed subjects, 13,100 controls). The results showed an association between the depression of the elderly and the risk of dementia of all types (OR 1.85 [95\% CI 1.67-2.04]), Alzheimer's dementia (OR 1.65 [95\% CI] 1.42-1.92]) and vascular dementia (OR 2.52 [95\% CI 1.77-3.59]). The risk was higher for vascular dementia than for Alzheimer's disease.

The weakness of these studies is related to the fact that in fact for most of the criteria taken into account, to the extent that the patients present symptoms of depression more than of depression clearly characterized.

\section{Depression or apathy}

Tegorical approach of depression concerns the links between depression and apathy. The biomedical approach, particularly in the field of cerebral / cognitive aging, is desperate to identify two distinct and homogeneous entities (two "diseases"), which would be defined by specific criteria. Recall that, according to the DSM 5, the identification of a "major depressive disorder" involves in particular the presence of at least one of the following two symptoms: a depressed mood or a loss of interest and pleasure. However, a recent characterization of apathy [12], identifies three types of apathetic manifestations: -the loss or reduction of goal-directed behavior (loss of self-initiated behavior and loss of environmentally stimulated behavior); -the loss or decrease of goal-directed cognitive activity (loss of spontaneous ideas and curiosity for routine and new events, loss of environmental-driven ideas and curiosity for routine and new events). - Loss or diminution of emotions observed or self-reported (loss of spontaneous, observed or self-reported emotion, loss of emotional reactivity to stimuli or positive or negative events).

In addition to the highly questionable character of the sharp distinction drawn between the spontaneous or reactive appearance of manifestations of apathy and the distinction between loss of goaldirected behavior and loss of goal directed cognitive activity (a loss of interest obviously being very much linked to a loss of initiation of the action and a problem of initiation of the action that can gradually lead to a loss of interest), we can only be struck by the importance the overlap between loss of interest and pleasure (one of the two required criteria of depression) and loss or decrease in goal-directed cognitive activity, as well as loss or diminution of emotions [13] . Moreover, each of the expressions of apathy may in fact refer to multiple factors, some of which are directly associated with depressive manifestations.For example, the loss or decline of goal-directed behavior may be underpinned by problems affecting different psychological factors.

Auctores Publishing - Volume1-10030 www.auctoresonline.org Page - 02
Action planning, prospective memory and multitasking, motivation of approach and avoidance, intrinsic motivation, projection into the future and anticipation of pleasure, evaluation of the effort to be allocated depending on the difficulty task, sense of self efficacy and control, etc. Many of these problems are also seen in "depression" and are modulated by a negative mood state. The same goes for the other two expressions of apathy [14]. It therefore seems completely pointless to distinguish, within aging, "apathy" and "depression" as distinct categories, since the manifestations grouped around these two entities and the factors associated with them overlap to a large extent. In addition, the use of these categories naturally leads to reducing the complexity of the factors underlying the various depressive and apathetic manifestations and to focusing primarily on neurobiological factors.

\section{Conclusion}

If we want to better understand the nature of the links between "depression" and problematic cognitive / cognitive aging, it seems more appropriate to adopt an approach that is based on the various symptoms that make up the "depression" entity. To identify the psychological mechanisms that underlie them, as well as the biological, social and eventrelated factors associated with them, from a continuum perspective. In study comparing healthy elderly volunteers and age-matched Alzheimer's dementia patients [15], there was no difference in the intensity of the symptoms of depression but it was shown that apathy was present in demented patients. So, there is often confusion in this type of patients between depression and apathy. Moreover, it remains true that a first episode of depression in patients over 70 years is correlated in $90 \%$ with the onset of Alzheimer's disease.

\section{References}

1. Bennett S, Thomas AJ (2014) Depression and dementia: cause, consequence or coincidence Maturities 79:184-190.

2. Barnes DE, Yaffe K (2011) The projected effect of risk factor reduction on Alzheimer's disease prevalence. Lancet Neurol 10:819828.

3. Enache D, Winblad B, Aarsland D (2011) Depression in dementia: epidemiology, mechanisms, and treatment. Curr Opin Psychiatry 24: 461-472.

4. Derouesné C, Lacomblez L (2004) [Depression and dementia]. Psychol Neuropsychiatr Vieil 2 Suppl 1: S35-42.

5. Steffens DC, Otey E, Alexopoulos GS, Butters MA, Cuthbert B, et al. (2006) Perspectives on depression, mild cognitive impairment, and cognitive decline. Arch Gen Psychiatry 63: 130-138.

6. Ganguli M (2009) Depression, cognitive impairment and dementia: Why should clinicians care about the web of causation? Indian $\mathbf{J}$ Psychiatry 51 Suppl 1: S29-34.

7. 7. Hermida AP, McDonald WM, Steenland K, Levey A (2012) The association between late-life depression, mild cognitive impairment and dementia: is infl ammation the missing link? Expert Rev Neurother 12: 1339-1350.

8. Lyketsos CG (2010) The interface between depression and dementia: where are we with this important frontier? Am J Geriatr Psychiatry 18:95-97.

9. Ownby RL, Crocco E, Acevedo A, John V, Loewenstein D (2006) Depression and risk for Alzheimer disease: systematic review, metaanalysis, and metaregression analysis. Arch Gen Psychiatry 63: 530538.

10. Jorm AF (2001) History of depression as a risk factor for dementia: an updated review. Aust N Z J Psychiatry 35: 776-781.

11. Diniz BS, Butters MA, Albert SM, Dew MA, Reynolds CF (2013) Latelife depression and risk of vascular dementia and Alzheimer's disease: systematic review and meta-analysis of community based cohort studies. Br J Psychiatry J Ment Sci 202: 329-335.

12. Mulin E, Zeitzer JM, Friedman L, Le Duff F, Yesavage J, et al. (2011) Relationship between apathy and sleep disturbance in mild and moderate Alzheimer's disease: an actigraphic study. J Alzheimer's Dis 25: 85-91. 
13. Robert PH, Mulin E, Malléa P, David R (2010) REVIEW: Apathy diagnosis, assessment, and treatment in Alzheimer's disease. CNS Neurosci Ther 16: 263-271.

14. Radakovic R, Starr JM, Abrahams S (2017) A Novel Assessment and Profi ling of Multidimensional Apathy in Alzheimer's disease. J Alzheimer's Dis 60: 57- 67.
15. Vercelletto M, Martinez F, Lanier S, Magne C, Jaulin P, et al. (2002) Negative symptoms, depression and Alzheimer's disease. Int $\mathrm{J}$ Geriatr Psychiatry 17: 383-387. 